\title{
Bromatological Analysis of the Mojicón for Greater Consumption in the City of Riobamba
}

\section{Análisis Bromatológico de Mojicón Para su Mayor Consumo en la Ciudad de Riobamba}

I International Seminar of Livestock and Agroindustrial Production ESPOCH 2020

Corresponding Author:

M. Bravo

belen_b33@hotmail.com

Published: 2 September 2021

Production and Hosting by

Knowledge E

(c) K. Amboya et al. This article is distributed under the terms of the Creative Commons Attribution License, which permits unrestricted use and redistribution provided that the original author and source are credited.
S OPEN ACCESS

\author{
K. Amboya ${ }^{1}$, R. Licuy ${ }^{2}$, and M. Bravo ${ }^{3}$ \\ ${ }^{1}$ Estudiante, Facultad de Ciencias Pecuarias, Escuela Superior Politécnica de Chimborazo, \\ Riobamba, Ecuador \\ ${ }^{2}$ Pasante, Productos San \\ ${ }^{3}$ Docente, Escuela Superior Politécnica del Chimborazo, Riobamba, Ecuador
}

\section{Abstract}

The mojicones are traditional bread dough products that are very popular in several provinces of the central area of the Sierra. They are desired for their sweet taste and their benefits when consumed in the daily diet. This project sought to understand the bromatological composition of the mijicon to improve its commercialization in the city of Riobamba. A descriptive nonexperimental design methodology was used with a correlational approach. According to the bromatological analysis, the composition in a $100 \mathrm{~g}$ portion was as follows: protein (7.92\%), fat (7.49\%), total dietary fiber (5.37\%), carbohydrates (73.2\%), sodium chloride $(6,762.22 \mathrm{mg})$, total sugars (348.7 mg), sodium (265.87\%), moisture (4.45\%) and ashes (1.44\%). An informal survey was carried out through various social networks to examine the acceptability of the product and its pros and cons; a SWOT analysis was used. The results showed that the product has high acceptability because it is an innovative product, and its disadvantage is that capital is too weak to stock up on its advertising and marketing. It is therefore possible to recommend wider consumption of the product by a larger population, due to its substantial energy and nutritional benefits.

Keywords: mojicon, bromatological, acceptability, demand, non-experimental design.

\section{Resumen}

Los mojicones son productos de masa pan, muy populares por tradición en varias provincias de la zona central de la sierra, siendo apetecido por personas que le gusta el sabor dulce, conociendo de su beneficio al consumirlo en la dieta diaria. La presente investigación busca conocer la composición bromatológica del mojicón y de este modo mejorar su comercialización en la ciudad de Riobamba, se uso una metodología de diseño no experimental de carácter descriptivo con un enfoque correlacional. En el análisis bromatológico se obtuvo los siguientes resultados, la proteína (7,92\%), la grasa (7,49\%), fibra dietética total (5,37\%), carbohidratos (73,2\%), cloruro de sodio $(6.762,22 \mathrm{mg})$, azucares totales $(348,7 \mathrm{mg})$, sodio $(265,87 \%)$, humedad $(4,45 \%)$, cenizas $(1,44 \%)$ en una proporción $100 \mathrm{~g}$, siendo importante compararla con otros productos de características similares. Para la aceptabilidad del producto se la realizo una encuesta informal por diversas redes sociales, a la vez determinando los pros y contras del producto siendo sustancial determinar un análisis FODA. Entonces debido al gran aporte energético como nutricional este producto puede ser consumido por una mayor población, teniendo una buena aceptabilidad del producto por ser un producto innovador, teniendo como desventaja de un capital débil para abastecerse de publicidad y mercadeo. 


\section{Introducción}

Los mojicones son bizcochos elaborados generalmente con masa que se corta en pequeñas porciones y se empapa o envuelve con panela [1]. El mojicón es un producto de masa pan conocido como dulce tradicional el cual no es muy comercializado ya que es un producto que solo se vende en la región Sierra del Ecuador. Los mojicones eran bastante apetecidos y consumidos por tradición por gente de años atrás, pero se busca recuperar la tradición de nuestros antepasados [2].

En el Ecuador y en la ciudad de Riobamba el consumo de galletas, dentro de los cuales se encuentran los mojicones, se ha convertido en uno de los sectores más dinámicos en el área de alimentos. En este sector se está generando una tendencia de beneficio funcional, donde se resalta la importancia de la salud, las fibras, los cereales y el bajo contenido de calorías [3].

La aceptabilidad del producto en la provincia de Tungurahua representa un $45 \%$ y en la provincia de Imbabura un 55\% [4]. Los productos y precios de producto actuales de similares características que se transforman en la competencia afectarán en la comercialización del producto objeto de esta investigación, en este caso el tipo de presentación y el valor nutricional de los mojicones influirán en su aceptabilidad y logrará maximizar su venta.

Los análisis bromatológicos son análisis físico-químicos de la materia que compone a los nutrientes, pues etimológicamente se puede definir a la Bromatología como Broma, 'alimento', y logos, 'tratado o estudio', es decir, que la bromatología es la ciencia que estudia los alimentos, sus características nutricionales [5].

La Asociación de Químicos Analíticos Oficiales también conocida como AOAC contribuye técnicas en la creación, validación y publicación mundial de métodos de ensayo analíticos fiables los que se utilizaran en esta investigación. Sus áreas de interés incluyen, pero no se limitan a, la seguridad de los alimentos, bebidas, suplementos dietéticos, fertilizantes, alimentos para animales, suelo y agua, y medicamentos veterinarios. El objetivo de los métodos de ensayo es evaluar la pureza de los materiales utilizados en la producción de los productos alimenticios, y sus ingredientes [6].

Con el desarrollo de esta investigación se busca conocer la composición bromatológica del mojicón, encontrar pro y contras del producto, y de este modo mejorar su comercialización en la ciudad de Riobamba. La aplicación del método de weende para obtener las características del producto permite resaltar las cualidades del producto, para que aprovechando estos beneficios se mejore su comercialización.

\section{Materiales y Métodos}

Esta investigación se aplica tanto cuantitativa como cualitativamente, ya que todas las variables a evaluarse pueden ser medibles mediante la toma de datos numéricos y la realización de pruebas a nivel de laboratorio, además debido a que se pueden desarrollar pruebas de análisis sensorial y aspectos referentes a la calidad del producto formulado [7]. 
La metodología utilizada en esta investigación fue un diseño experimental y no experimental, con una investigación de carácter descriptivo y correlacional, se estudiaron variables independiente y dependiente, también se utilizó la investigación aplicada fundamental, se empleó la investigación bibliográfica como: Artículos científicos, tesis, la información existente en bibliotecas, revistas entre otros métodos documentales [8].

Los tipos de investigación que intervienen en la elaboración la investigación son:

\subsection{Experimental}

Porque todo el análisis físico, químicos del producto formulado y terminado se elaborarán a nivel de laboratorio utilizando técnicas planteadas por las normas INEN con el fin de obtener datos reales que permitan determinar la calidad de los productos de bocaditos de pan envueltos en miel de panela (mojicones).

\subsection{Determinación de análisis proximal}

Los análisis proximales fueron aplicados a los mojicones (bizcochos envueltos en miel de panela), con una presentación diferente a la tradicional, una forma redonda y pequeña. La Proteína, Kjeldahl se determinó bajo las condiciones descritas por el método de referencia AOAC Ed 20, 2016 (2001.11) [9]. Para la determinación de Grasa, se utilizó la gravimetría, por el método de referencia AOAC Ed 20, 2016 (2003.06). Con respecto a la determinación de fibra dietética total enzimática, se empleó la gravimetría, con referencia a lo establecido por la AOAC Ed 20,2016. La determinación de carbohidratos totales y azucares totales en los mojicones, se determinó por el método de referencia AOAC 923.09 [10]. La determinación de cenizas se determinó con referencia en el método AOAC 923.09 [11].

\subsection{Determinación de la estabilidad de los mojicones}

Esta determinación se lo realizo en dos sesiones, una al primer día y la otra sesión después de 60 días. La determinación de la estabilidad se lo realizo referente a tres parámetros, los cuales fueron $\mathrm{pH}$, humedad y mohos. $\mathrm{El} \mathrm{pH}$, de los mojicones se determinó por potenciometría, con la ayuda de la norma INEN 521 [12]. Para la determinación de la humedad se lo realizó por gravimetría, bajo las referencias del método AOAC Ed 20, 2016 (925.10). Mientras que para la determinación de hongos en los mojicones se lo realizó por medio de Petrifilm, con el método AOAC Ed 20, 2016.

\subsection{Determinación del consumo de los mojicones}

Para poder determinar el panorama en cuanto a la competencia de los mojicones se realizará un análisis FODA, en donde se resalta las fortalezas, oportunidades, debilidades y amenazas de los mojicones. 
Se utilizaron técnicas de investigación de fuente primaria, las cuales fueron encuestas, a través de preguntas cerradas basadas en conocimientos empíricos, el método empleado fue el inductivo y el deductivo, con el fin de observar, analizar y clasificar los acontecimientos. El proyecto posee un carácter cualitativo - Descriptivo, se considera cualitativo, ya que estará fundamentado en la recolección de información; y descriptivo porque se realiza a través de la búsqueda de información relevante.

\section{Resultados}

\subsection{Determinación del análisis proximal}

Con respecto a la composición de los mojicones en lo que se refiere al análisis proximal los resultados se pueden observar en la Tabla 1. estos resultados permiten determinar el nivel de contenido de azúcar que tienen los mojicones, alto contenido de azúcar (rojo), medio en grasa (amarillo) y bajo en sal (verde), esto según la semaforización establecida por normas del Ecuador.

\section{Table 1}

Resultados del análisis proximal.

\begin{tabular}{|l|c|c|}
\hline \multicolumn{3}{|c|}{ RESULTADOS OBTENIDOS } \\
\hline Ensayo /Prueba & U nidades & Resultados \\
\hline Prote ina & $\%(\mathrm{Nx} 6.25)$ & 7.92 \\
\hline Grasa & $\%$ & 7.49 \\
\hline Fibra Dietética Total & $\%$ & 5.37 \\
\hline Carbohidratos & $\%$ & 73.2 \\
\hline Cloruro de sodio & $\mathrm{mg} / 100 \mathrm{~g}$ & 6762.22 \\
\hline AzucaresTotales & $\mathrm{g} / 100 \mathrm{~g}$ & 348.7 \\
\hline Sodio & $\mathrm{mg} / 100 \mathrm{~g}$ & 265.87 \\
\hline Humedad & $\%$ & 4.54 \\
\hline Cenizas & $\%$ & 1.44 \\
\hline
\end{tabular}

\subsection{Determinación de la estabilidad de los mojicones}

Los resultados de la determinación de la estabilidad de los mojicones, en las dos sesiones fueron favorables como se puede observar en Tabla 2. Según los resultados obtenidos, el periodo de vida útil de los mojicones es de 60 días.

A manera de resumen de todos los resultados de análisis bromatológico, se presenta en la Tabla 3, el valor o información Nutricional de los Mojicones.

Los resultados del FODA se presentan a continuación en la siguiente ilustración, en donde se resaltan los mayores puntos, y los que son pertinentes para este estudio son los fortaleza y oportunidades que tiene el mojicón en el mercado de la ciudad de Riobamba.

De acuerdo con las encuestas realizadas, se ha tomado un enfoque a dos preguntas, que una vez arrojado los datos correspondientes se tiene lo siguiente: 
Table 2

Resultados del estudio de estabilidad.

\begin{tabular}{|c|c|c|c|}
\hline \multicolumn{4}{|c|}{ Resultados del Estud io de Estabilidad } \\
\hline Envejecimiento: Normal al ambiente & Temperatura: $20 \pm 3^{\circ} \mathrm{C}$ & & \\
\hline Tiempo de estudio:60 dias & Fecha de inicio: 18 de Octubre de 2018 & & \\
\hline & Fecha de Finallzacion: 19 de diciembre de 2018 & & \\
\hline Ensayos solicitados & Unidades & Primer Control de estabilidad Dia1 & Segundo Control de Estabilidad Dia 60 \\
\hline $\mathrm{pH}$ & Unidades de $\mathrm{pH}$ & 5.62 & 5.64 \\
\hline Humedad & $\%$ & 4.54 & 5.04 \\
\hline Mohos & $\mathrm{UFC} / \mathrm{g}$ & $<10$ & $<10$ \\
\hline
\end{tabular}

Table 3

Información nutricional.

\begin{tabular}{|c|c|c|}
\hline \multicolumn{3}{|c|}{ Cantidad por envase: $180 \mathrm{~g}$} \\
\hline \multicolumn{3}{|l|}{ Tamaño por porción: $15 \mathrm{~g}$} \\
\hline \multicolumn{3}{|l|}{ Porciones por envase: 12} \\
\hline Cantidad por porción & & $\%$ Valor diario \\
\hline Energías (calorías): & 59 kcal & $1431 \mathrm{~kJ}$ \\
\hline calorías de la grasa: & $10 \mathrm{kcal}$ & 1 \\
\hline Grasa & $1 \mathrm{~g}$ & 1 \\
\hline Ácidos grasos saturados & $7 \mathrm{~g}$ & 36 \\
\hline $\begin{array}{l}\text { Ácidos grasos } \\
\text { monoinsaturados }\end{array}$ & $4 \mathrm{~g}$ & \\
\hline $\begin{array}{l}\text { Ácidos grasos } \\
\text { Poliinsaturados }\end{array}$ & $4 \mathrm{~g}$ & \\
\hline Ácidos grasos trans. & $0 \mathrm{~g}$ & \\
\hline Colesterol & $2 \mathrm{mg}$ & 1 \\
\hline Sodio & $40 \mathrm{mg}$ & 2 \\
\hline Carbohidratos totales & $11 \mathrm{mg}$ & 4 \\
\hline Fibra dietética & $1 \mathrm{~g}$ & 3 \\
\hline Azúcares totales & $52 \mathrm{~g}$ & \\
\hline Proteína & $1 \mathrm{~g}$ & 2 \\
\hline
\end{tabular}

Se entiende que se obtuvo un resultado favorable en cuanto a la compra de mojicones puesto a que la población conoce de este producto por su vista llamativa y dulzor con un $68 \%$ mientras que el $32 \%$ de la población encuestada no comprarían el producto por problemas de salud relacionados con el azúcar y por otro lado no lo conocen debido a que no se comercializa en todas las ciudades del país, ya que este alimento es considerado como un producto tradicional de la ciudad de Riobamba. 


\section{Table 4}

Análisis FODA.

\section{Análisis FODA}

\section{Fortaleza}

- El producto es innovador.

- El producto cumple con los parámetros de calidad e inocuidad.

- Es un producto rico en energía por sus azucares que lo contiene.

- La materia prima es fácil de adquirir

- Se pretende disminuir la proporción la proporción de azucares.

\section{Debilidades}

- Posible apatía del consumidor hacia el producto, sobre todo para los celiacos.

- Al ser una empresa nueva no tenemos experiencia en el rubro y no vamos a obtener grandes ganancias.

- No contamos con un capital suficiente por lo que nos veremos obligados a realizar todos los gastos por nuestra cuenta.

- Aun no somos un negocio conocido en el mercado.

\section{Oportunidades}

- Debido a la preocupación de las personas para consumir alimentos saludables se caracterizan por estar abiertos aprobar nuevos productos y sabores, lo cual. puede favorecer la venta de nuestro producto.

- Cubrir demanda insatisfecha con un producto que cuide la salud.

- Aprovechar la obtención de materia prima disponible.

- Difundir nuestro producto en los medios de comunicación y ganar reconocimiento.

- Acceder a la tecnología de alta calidad para mejorar la producción.

\section{Amenaza}

- La existencia de los competidores en el mercado que se dedican a la misma actividad y la presencia de productos alternativos.

- Competencia posicionada en el mercado, pueden abaratar costos para tratar de ofrecer precios más competitivos y absorber la microempresa.

- Competencia desleal, que se copie la idea de negocio.

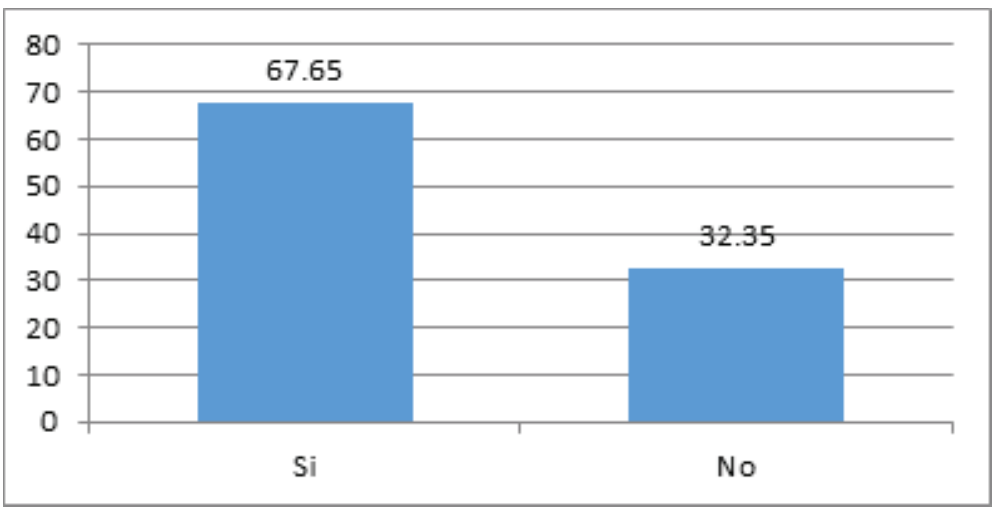

Figure 1

Análisis de ventas.

\section{Discusión}

\subsection{Análisis proximal}

Se ha relacionado dos tipos de galletas en la cual, la primera según [13] una galleta (A) dentro de su composición tiene el $10,25 \%$ de proteína, el $20,17 \%$ de grasa y $42,90 \%$ de carbohidratos en una porción de $100 \mathrm{~g}$. En cambio [14] la galleta (B) dentro de su 
composición tiene el $8,8 \%$ de proteína, el $15 \%$ de grasa y $6,50 \%$ de carbohidratos en una proporción de $37 \mathrm{~g}$. Al comparar con el resultado nuestro producto del análisis proximal realizado, en la Tabla 1, se puede observar que el porcentaje de proteínas es de $7,92 \%$, grasa de $7,49 \%$ y carbohidratos $73,2 \%$ en el producto formulado.

Entonces podemos aseverar que los mojicones presentan niveles adecuados para suplementar la dieta, y son más seguros y aptos para el consumo humano ya que presentan un porcentaje de grasa menor en comparación con las galletas, gracias a los carbohidratos generara más energía a las personas que lo consuman y por el contenido de proteínas que está en rango de normas establecidas relacionado con las comparaciones realizadas, siendo importante ya que ayudará a mantener un buen estado de salud.

Investigaciones demuestran que existe una estrecha relación entre la vida útil y la evaluación sensorial de muchos alimentos [15], además en galletas (productos similares), este parámetro está definido por el cambio de sus propiedades sensoriales, afirmando así mediante pruebas realizadas que el tiempo de vida útil de las galletas es de mayor a los 21 días desde el día cero el cual es el de su elaboración [16], el cambio que las galletas de tipo wafle que estas pueden tener un tiempo de vida útil igual a 3 meses durante temperatura ambiente realizando una determinación de contenido de humedad cada semana el cual ha tenido un pequeño incremento sin alterar ni afectar sus propiedades. En relación a nuestro producto mojicón podemos ver que el tiempo de vida útil es muy bueno correspondiente a 60 días superando a las galletas normales pero inferior que el de galletas wafles, pero esto también depende mucho de la composición de esta galletas wafles aclarando así que los mojicones tienen un tiempo de vida útil larga en comparación de productos tradicionales como las galletas [17]. El porcentaje de humedad de nuestro producto es de 5,40\% a los 60 días a cuál el máximo es de $10 \%$ de acuerdo a la norma INEN 20185:2005, esto a la vez potenciará como producto que tiene mayor tiempo de vida útil por su baja cantidad humedad [18].

Varias investigaciones mencionan que en la mayoría de los casos se podría pensar que los consumidores eligen sus productos tomando en cuenta el precio y la marca, sin embargo, existen otros motivos que llevan a una persona a elegir un producto respecto a otro. A pesar de que los consumidores se comporten de formas diferentes y tengan diferentes actitudes y comportamientos respecto a la nutrición, los grupos más grandes de consumidores demandan altos estándares de seguridad y mayores méritos nutricionales en los alimentos [19, 20].

Uno de los principales factores que los consumidores toman en cuenta al elegir un producto alimenticio se debe a que presenten propiedades saludables y un sabor agradable por lo que la mayoría de ellos se fijan siempre en la información nutricional de los alimentos, estos se fijan principalmente en la cantidad de grasas saturadas, que tengan menos aditivos, bajas cantidades de sal, con más fibra y calcio por lo que estos productos son los más demandados por los clientes [17]. Al realizar el análisis bromatológico de los mojicones y darles a conocer esta información a los consumidores, estos se vieron más interesados en adquirir el producto debido a que presenta baja cantidad de sal y grasa, al mismo tiempo lo asociaron con un alimento saludable que 
presenta un buen sabor y por lo tanto lo compraron de forma inmediata y de esta manera aumentaron las ventas de los mojicones.

\section{Conclusiones}

Debido a que es un producto con grandes beneficios tanto energéticos como nutricionales este producto puede ser consumido por mayor población.

Se determinó los condiciones favorables y adversas para su distribución, lo cual nuestro producto tuvo una gran acogida ya que se consideró un producto innovador, teniendo una desventaja sobre un capital débil para poder mejor su publicidad y mercadeo.

\section{References}

[1] Pérez M. Golosolandia. Pressreader. 2005 June 4. Available from: https://www.pressreader.com/ ecuador/el-universo/20190407/282252371903860.

[2] Pinto W, Ortega M. Deleita con el tradicional pan de trigo y maíz. El Universo. 2009 April 7. Available from: https://www.pressreader.com/ecuador/el-universo/20190407/282252371903860..

[3] Norma Técnica Obligatoria Nicaragüense. Panela, tapa de dulce y panela granulada (dulce granulado). Requisitos. Nicaragua: ICS; 2017.

[4] Melo R. 2016. El Telégrafo. 2016 February 7. Available from https://www.eltelegrafo.com.ec/noticias/ regional/1/los-dulces-del-norte-se-resisten-a-desaparecer.

[5] Fernández A. 2016. Evaluación fisicoquímica, sensorial y vida útil de galletas enriquecidas con subproductos proteicos de suero de quesería. 2016. Available from: https://www.redalyc.org/pdf/959/ 95945988003.pdf.

[6] FAO. Datos de composición de alimentos. Roma: FAO; 2006.

[7] CODEX 234. Métodos de análisis y de muestreo recomendados. 1999.

[8] Garay J, Navarro M. Análisis Bromatológico y microbiológicos de galletas. Bistream:; 2019.

[9] Chirinos W, Vargas N. Análisis proximal de galletas de harina de trigo. Revista Centro azúcar

[10] Latimer G. Official methods of analisis. OAC Internacional; 2016.

[11] Logroño M, Vallejo L. Análisis Bromatológico, sensorial y aceptabilidad de galletas. Revista de la Asociación Colombiana de Ciencia y Tecnología de los alimentos. 2015;63-64.

[12] Norma Técnica Ecuatoriana 521. Harinas de origen vegetal: Determinación de la acidez titulable. Quito: s.n.; 2012.

[13] Salazar G. Análisis bromatológicos. 2017. Academia.edu. [En línea] 25 de mayo de 2017. [Citado el: 22 de julio de 2020.] https://www.academia.edu/9256683/Analisis_Brimatologicos.

[14] Godoy R. Analisis, evaluacion sensorial y valor proteico de una galleta de harina de trigo (Triticum aestivum) y Harina de arveja dulce (Pisum sativum). Guatemala: Universidad de San Carlos de Guatemala; 2010.

[15] Estrella E. El pan de América: Etnohistoria de los alimentos aborígenes en el Ecuador. Quito: FUNDACYT; 1998.

[16] Méndez A, García D. Evaluación de galletas dulces tipo wafer a base de harina de arracha. Redalyc. 2007 December 17. Available from: https://www.redalyc.org/pdf/1799/179914078020.pdf..

[17] Gavilanez J. Galletas con base en concentraciones de harina de camote y maíz en el cantón Pichincha. Los Ríos: Universidad Técnica Estatal de Quevedo; 2017.

[18] Logrono M, Benítiz L. Análisis Bromatológico, sensorial y aceptabilidad de galletas y bebida nutritiva a base de una mezcla de quinua, arveja, zanahoria y tocte. Alimentos hoy. 2015;23:53-64.

[19] Sepúlveda S, Chavarria H. Factores no económicos de la competitividad. Coronado: IICA; 2001.

[20] CEOACCU. Confederación española de organización de amas de casa, consumidores y usuarios. 2015 March 23. Available from: https://www.mesaparticipacion.com/files/34_150909_Nuevos_h_bitos_de_ Compra_y_Consumo_de_Alimentos_2015.pdf. 